Supporting Information

\title{
Efficient Addition of Desired Carboxylate Ligands to CdSe Quantum Dots Passivated with Phosphonic Acids
}

Dong-Won Jeong, Tae-Hyeon Park, Jung Ho Lee and Du-Jeon Jang*

Department of Chemistry, Seoul National University, Seoul 08826, Korea

* Corresponding author. E-mail address: djjang@snu.ac.kr. 


\section{Index}

Characterization

page $\mathrm{S} 3$

Figure S1. TEM and STEM images

Table S1. Mean radii and stardard deviations

Figure S2. XRD patterns

Figure S3. XPS data

Figure S4. NMR spectra

Figure S5. In-situ NMR spectra

Figure S6. Absorption/PL spectra and emission decay profiles

Table S2. Emission decay constants

Figure S7. Crystal structure of wurtzite CdSe

Figure S8. Schematic of exciton dynamics

Analysis S1. Detail of exciton dynamics

Calculation S1. Binding amount of Z-type ligands

Figure S9. Structural model of CdSe QD

Calculation S2. Ligand composition of QDs

Figure S10. NMR spectrum with integrals of protons 


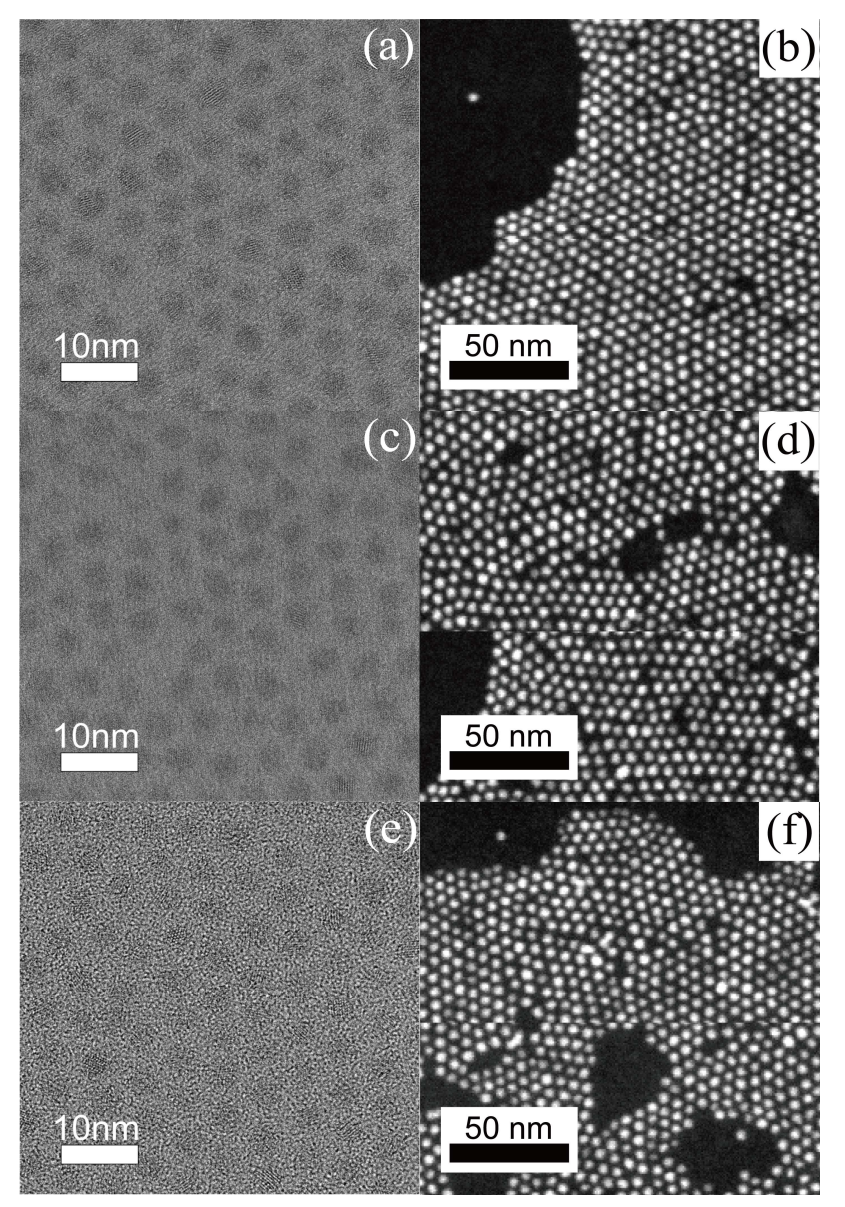

Figure S1. TEM (left) and STEM images (right) of CdSe QDs (a,b), CdSe QDs + OA (c,d), and CdSe $\mathrm{QDs}+\mathrm{Cd}(\mathrm{OA})_{2}(\mathrm{e}, \mathrm{f})$.

Table S1. Mean Radii and Stardard Deviations of QDs Measured from FE-TEM Images

$\begin{array}{cccc}\text { sample } & \text { CdSe QDs } & \text { CdSe QDs + OA } & \text { CdSe QDs + Cd(OA) } \\ \text { mean radius }(\mathrm{nm}) & 3.026 & 3.029 & 3.112 \\ \text { standard deviation }(\mathrm{nm}) & 0.175(5.78 \%)^{*} & 0.203(6.70 \%) & 0.202(5.1 \%)\end{array}$

*Relative standard deviation percentage.

Method. The field emission transmission electron microscope (FE-TEM) images were converted to FFT patterns using the Gatan Microscopy Suite 3 software. Patterns except QDs were masked, and converted back (Inverse FFT) to extract clear images. The ImageJ software (ver. 1.53e) was used to analyze the sizes of the QDs. After adjusting the threshhold using a triangle algorithm to convert each image into a binary image, nanoparticles having circularity over 0.85 and sizes between $5 \sim 10 \mathrm{~nm}^{2}$ were sampled. The average diameter was calculated from the binary images of the sampled nanoparticles. 


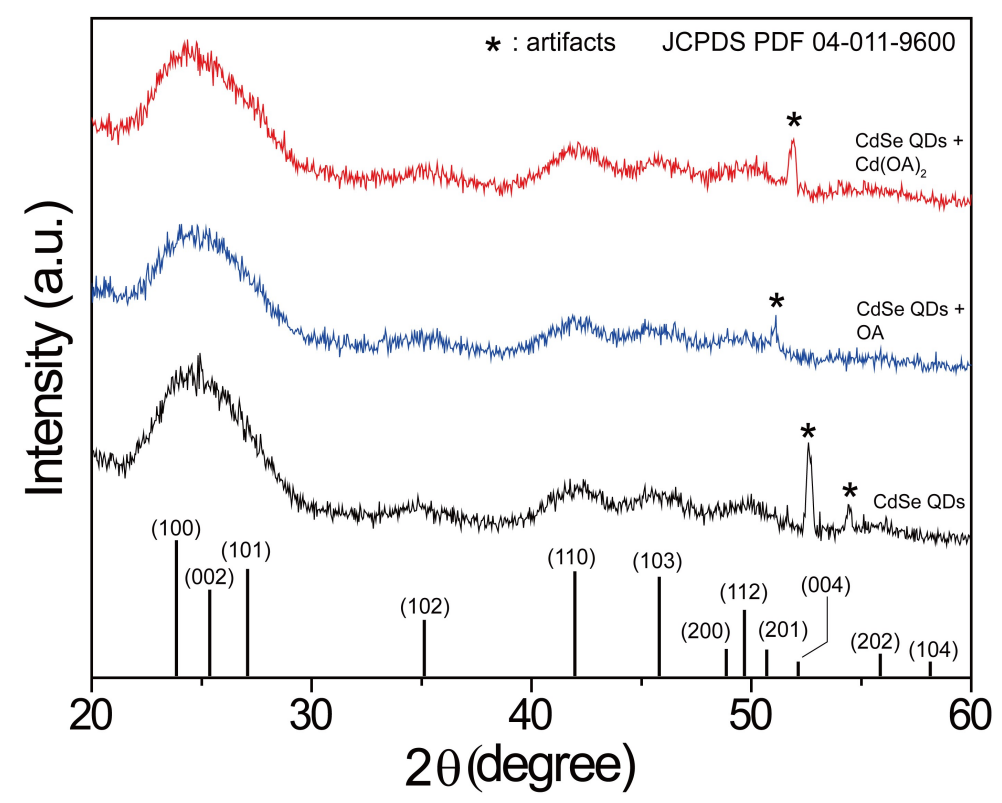

Figure S2. Grazing incidence X-ray diffraction (GI-XRD) patterns of CdSe QDs (black), CdSe QDs + $\mathrm{OA}$ (red), and CdSe QDs $+\mathrm{Cd}(\mathrm{OA})_{2}$ (blue). The pattern of each sample is consistent with that of the CdSe wurtzite structure (bottom). Artifacts appeared due to silicon wafer substrates.

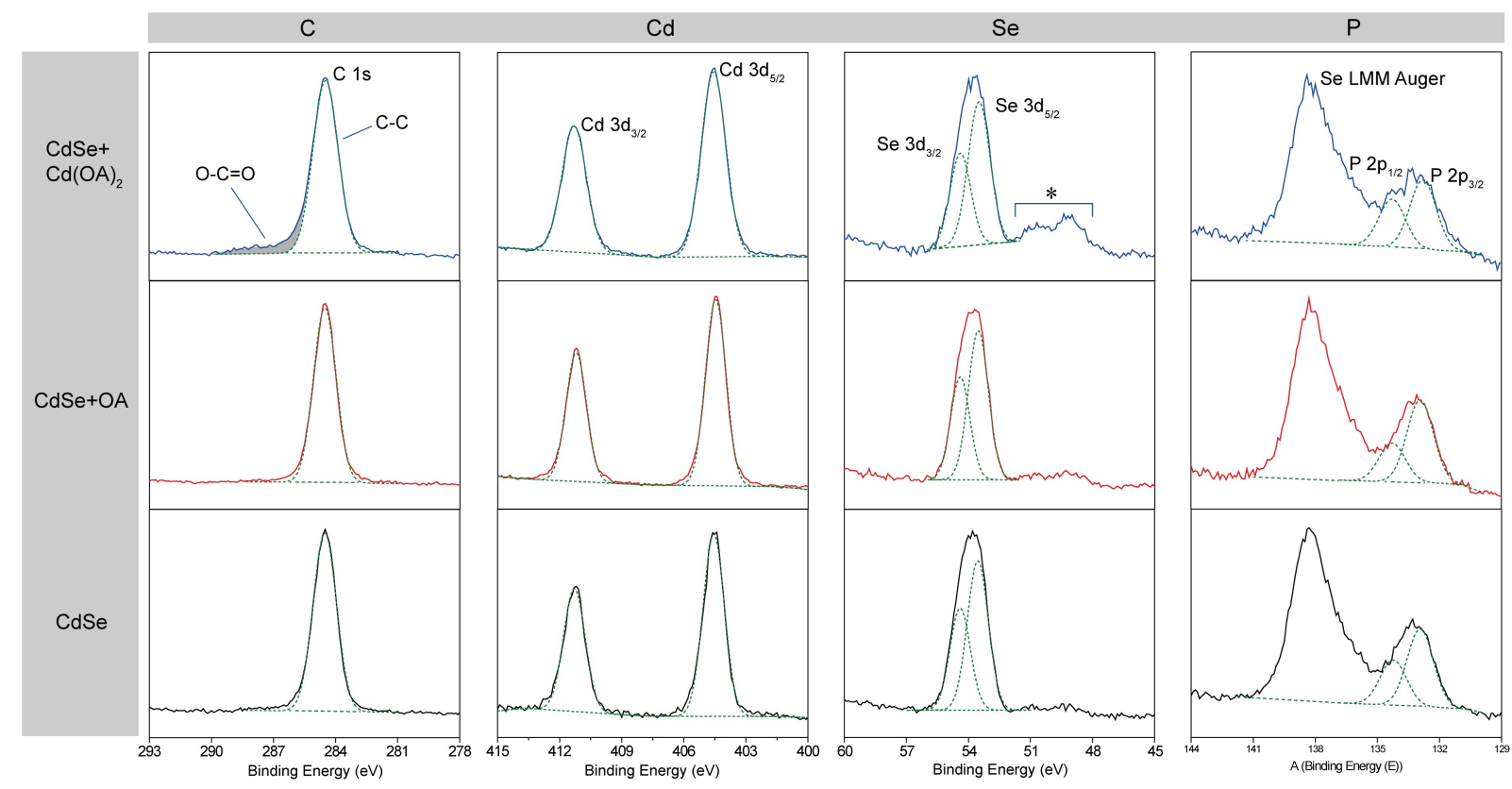

Figure S3. X-ray photoelectron spectroscopy (XPS) data of indicated samples. Note that the peaks next to selenium $3 \mathrm{~d}_{5 / 2}(*)$ are artifacts. 

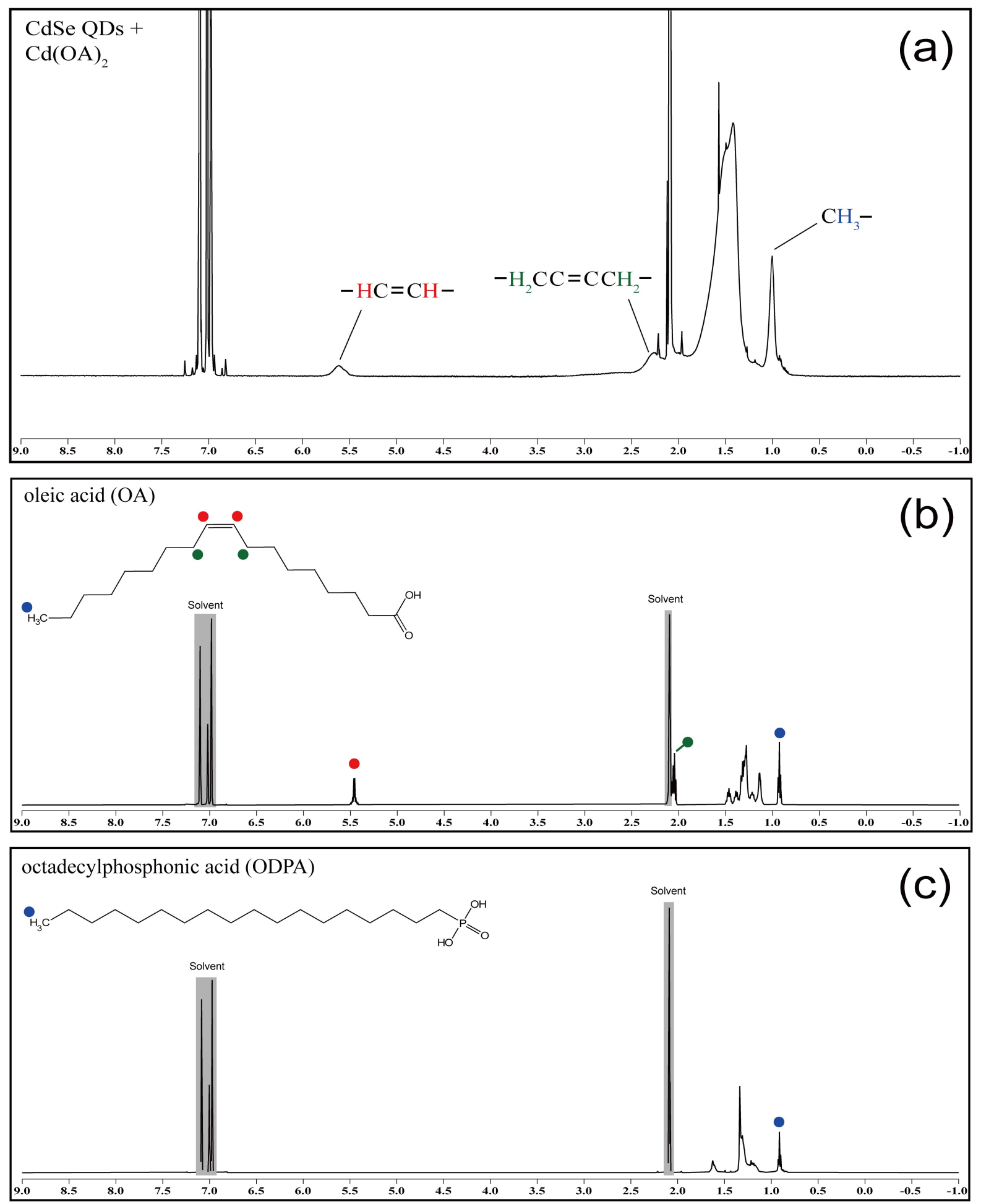

Figure S4. ${ }^{1} \mathrm{H}$ NMR spectra of the $\mathrm{CdSe}+\mathrm{Cd}(\mathrm{OA})_{2}$ sample (a), OA (b) and ODPA (c). OA and ODPA shown in NMR spectra are ligands used for the CdSe QD synthesis. Olefinic protons (red) and allylic protons (green) are the distinct aspects of OA compared to ODPA. However, both ligands have protons (blue) from the terminal carbon at the same position. 

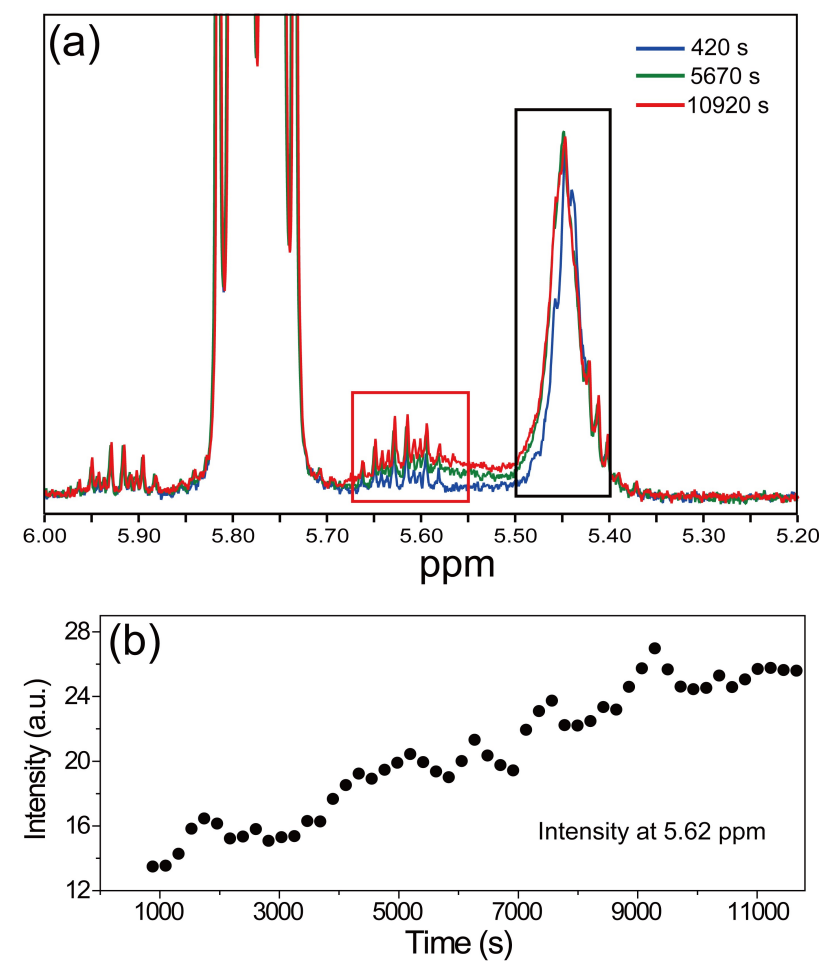

Figure S5. In-situ NMR spectra of a solution containing CdSe QDs $+\mathrm{Cd}(\mathrm{OA})_{2}$ (a) and variation of NMR peak intensity at $5.62 \mathrm{ppm}$ with time (b). The red square in (a) shows the olefinic protons of OA bound to CdSe QDs while the black square in (a) shows olefinic protons from $\mathrm{ODE}\left(\right.$ solvent), $\mathrm{Cd}(\mathrm{OA})_{2}$, and free OA. Note that the large peak at $5.77 \mathrm{ppm}$ corresponds to 1-octadecene. Although the $5.62 \mathrm{ppm}$ peaks of the sample were disturbed by heteronuclear coupling $\left({ }^{13} \mathrm{C}\right)$ from the $5.77 \mathrm{ppm}$ peak, there was no difficulty in recognizing the trend. 

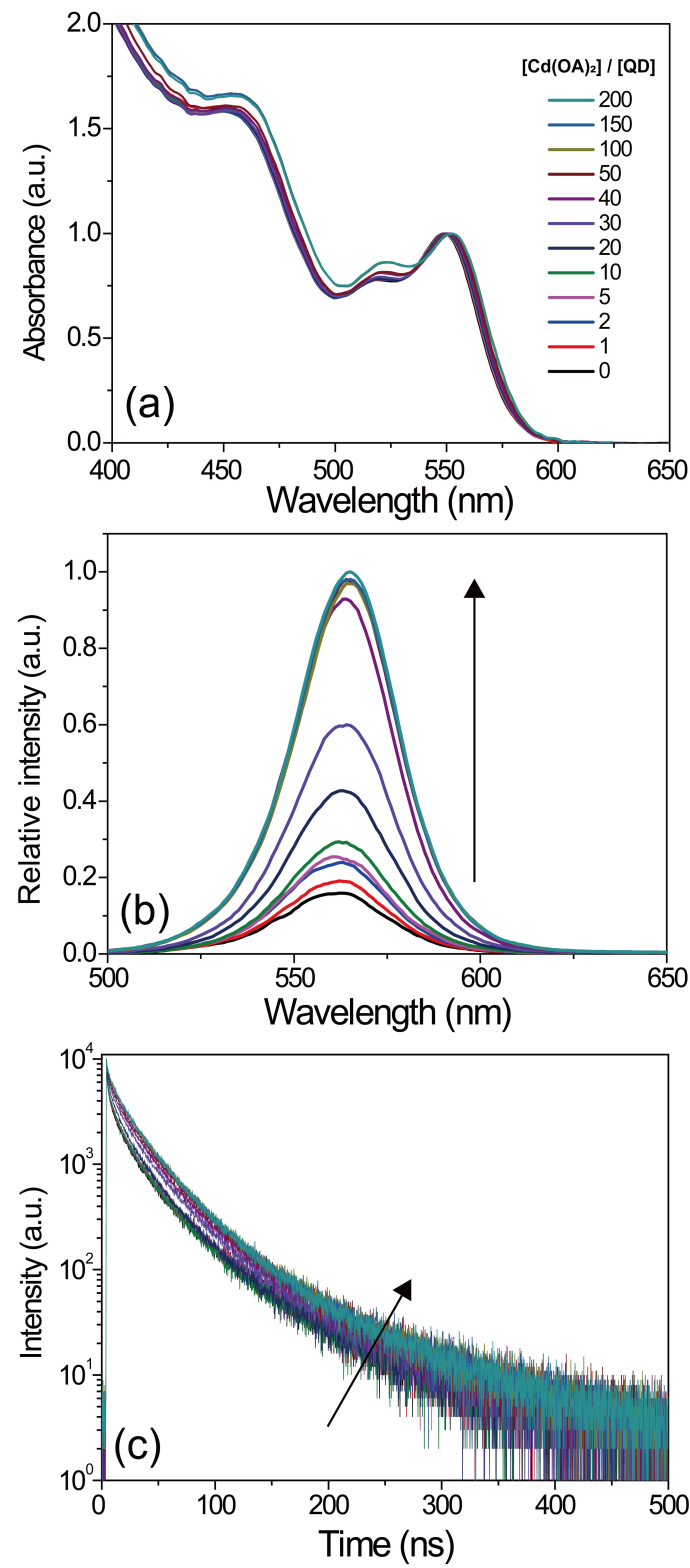

Figure S6. $\left[\mathrm{Cd}(\mathrm{OA})_{2}\right] /[\mathrm{QD}]$-dependent normalized-absorption (a) and PL (b) spectra and emission decay profiles (c). All the samples were synthesized via the same procedure except the amounts of added $\mathrm{Cd}(\mathrm{OA})_{2}$.

Table S2. Emission Decay Constants of CdSe QDs Samples

$\begin{array}{cccc}\text { sample } & \begin{array}{c}\text { relative initial } \\ \text { intensity }\end{array} & \text { decay time }(\mathrm{ns}) & \begin{array}{c}\text { mean lifetime } \\ (\mathrm{ns})\end{array} \\ \text { CdSe QDs } & 0.67 & 2.2(75.7 \%)+13.0(16.6 \%)+44.3(7.7 \%)^{\mathrm{a}} & 7 \\ \mathrm{CdSe} \text { QDs }+ \text { OA } & 0.71 & 2.2(75.7 \%)+13.0(16.6 \%)+44.3(7.7 \%) & 7 \\ \mathrm{CdSe} \text { QDs }+\mathrm{Cd}(\mathrm{OA})_{2} & 1.00 & 2.3(24.7 \%)+14.3(42.2 \%)+42.2(33.1 \%) & 21\end{array}$

a Initial amplitude percentage of each component. 

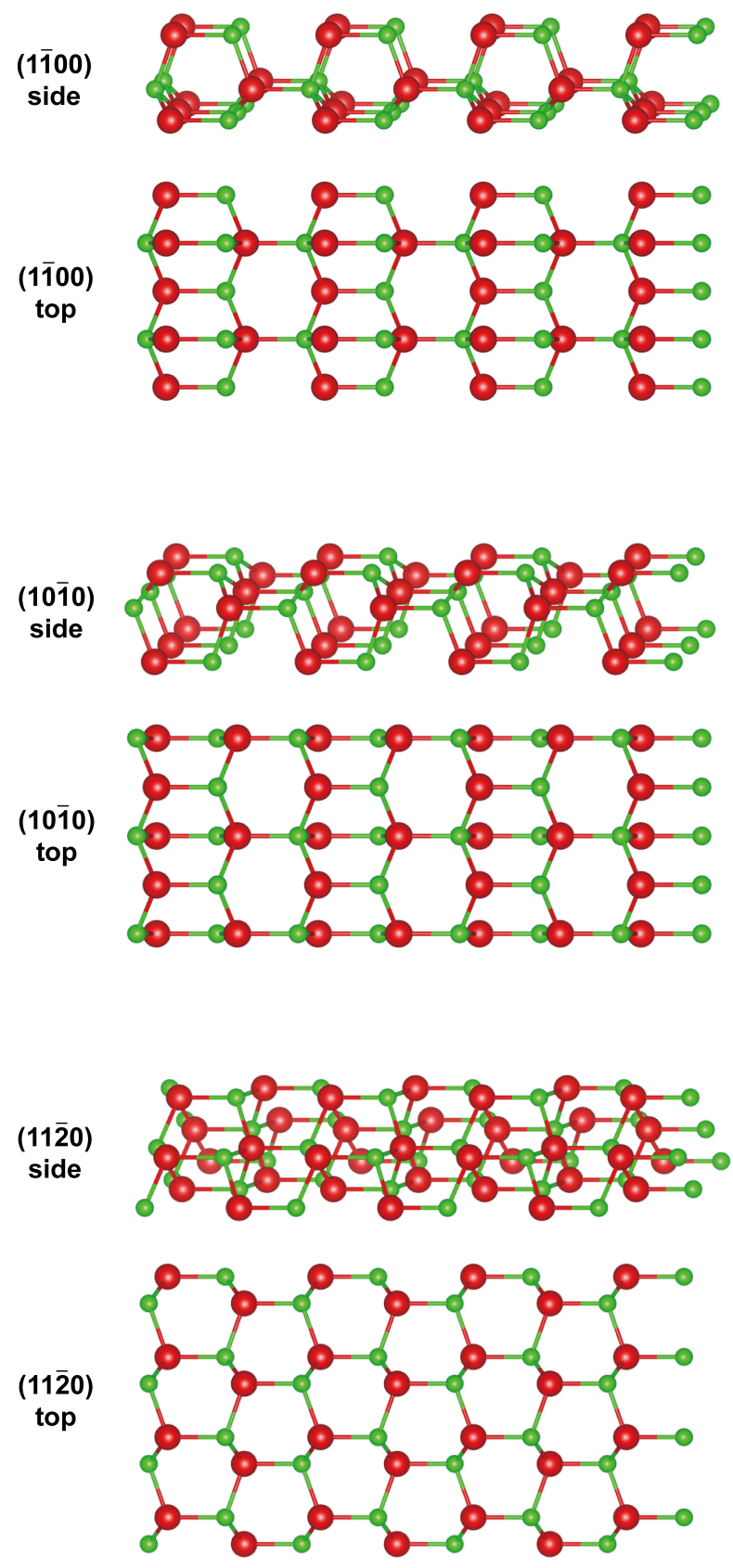

Figure S7. Illustration of three nonpolar facets of the wurtzite CdSe crystal. The red sphere represents cadmium and the green sphere represents selenium. Only (1100) has dicoordinated selenide. The other two facets have tricoordinated selenide which has no reduction effect on the PL of wurtzite CdSe QDs. 


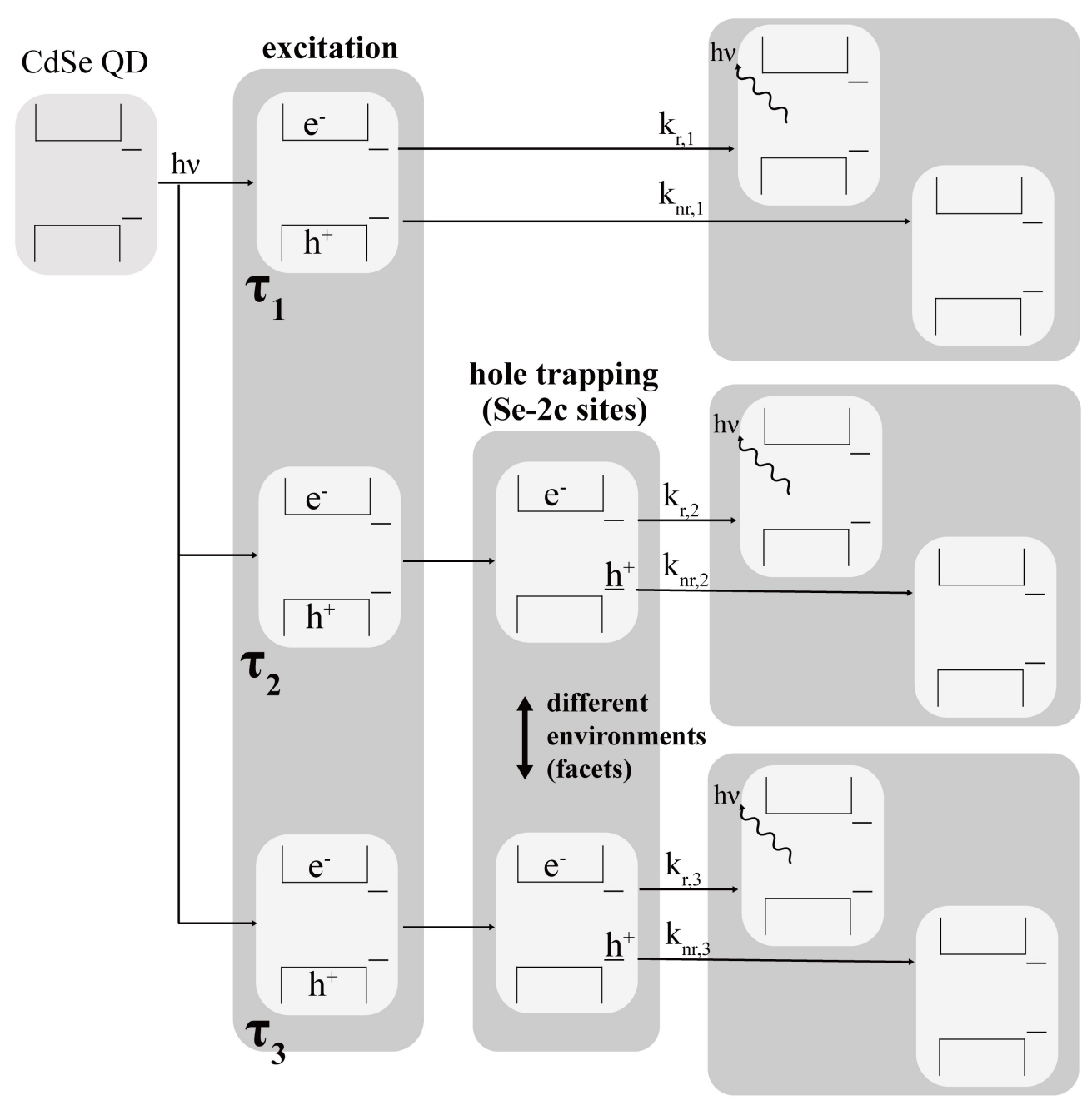

Figure S8. Overview of exciton decay pathways in QDs. Note that this schematic is simplified to explain our triexponential fitting according to ref. 41.

Analysis S1. We have fitted the PL decay profile of the CdSe QDs as a triexponential function. The exciton dynamics scheme of our synthesized QDs is shown in Figure S8. In this work, we have tried to correlate QD surface structures to time-resolved PL data by presenting an appropriate exciton dynamics model: Excitons undergo radiative decay and interact with trap sites in QDs at the same time. The nonradiative decay processes of excitons including multicarrier processes compete with radiative processes. Many groups have studied the spectroscopy of a single QD having a core-shell structure. It has been observed that nonradiative relaxation induced from multicarrier processes is dominant due to inorganic shell passivation. ${ }^{21-23}$ However, in the case of this study observing core QDs only, phonon- 
mediated nonradiative decay is more active than in QDs with inorganic shells, making their decay profiles complex. Therefore, it is reasonable to explain the PL decay constants with competition between radiative and nonradiative recombination processes as shown in eq S1:

$$
\tau_{1}=\frac{1}{k_{r, 1}+k_{n r, 1}} \quad \tau_{2}=\frac{1}{k_{r, 2}+k_{n r, 2}} \quad \tau_{3}=\frac{1}{k_{r, 3}+k_{n r, 3}}
$$

According to the transient absorption study of CdSe QDs in the visible and the NIR regions, both the longest and the second longest PL lifetimes have 100/0 for the value of \%electron / \%hole, which is the relaxation contribution ratio of $\mathrm{e}^{-/} \mathrm{h}^{+}$. Meanwhile, the decay with the shortest lifetime in the nanosecond region has $85 / 15$ for the value of \%electron / \%hole. ${ }^{41}$ In other words, electron dominant processes without hole contribution lead recombination in QDs, and these phenomena have been found ${ }^{41,48,49}$ to take place because of ultrafast hole trapping.

Holes and electrons relax rapidly to the respective lowest energy states of the QDs after excitation. ${ }^{48,49}$ At this time, the transient population is divided into a case 1 (all the holes fall into the trap sites) and a case 2 (hole trapping occurs partially). These intraband relaxation pathways make a difference in hole contribution to transient population. In our experiment, the decay profiles of $\tau_{2}$ and $\tau_{3}$ are generated from hole trapping, so there is no hole contribution. Meanwhile, the shortest time constant $\tau_{1}$ has partial hole trapping, having hole population. We assumed that dicoordinated selenides in QDs, which can act as localized hole traps, may trap all the holes at an ultrafast rate, whereas surface structures in QDs that are barely relevant to PL act as traps, resulting in partial hole trapping. 


\section{Calcuation S1. The binding amount of Z-type ligands on the CdSe QD surface.}

To verify atomic ratios of QDs, we introduce the QD structure model of the Weiss group. ${ }^{52}$ Although the QDs show a clear wurtzite structure in the XRD pattern, only the core, not the shell part, corresponds to the bulk wurtzite structure in this model. In other words, the core structure with the wurtzite CdSe bulk structure has 1 for the ratio of $\mathrm{Cd} / \mathrm{Se}$, and the shell structure of QDs has over 1 for the value of $\mathrm{Cd} / \mathrm{Se}$, meaning that extra $\mathrm{Cd}$ atoms are deposited on the surface of QDs as described this study (Figure S9a).
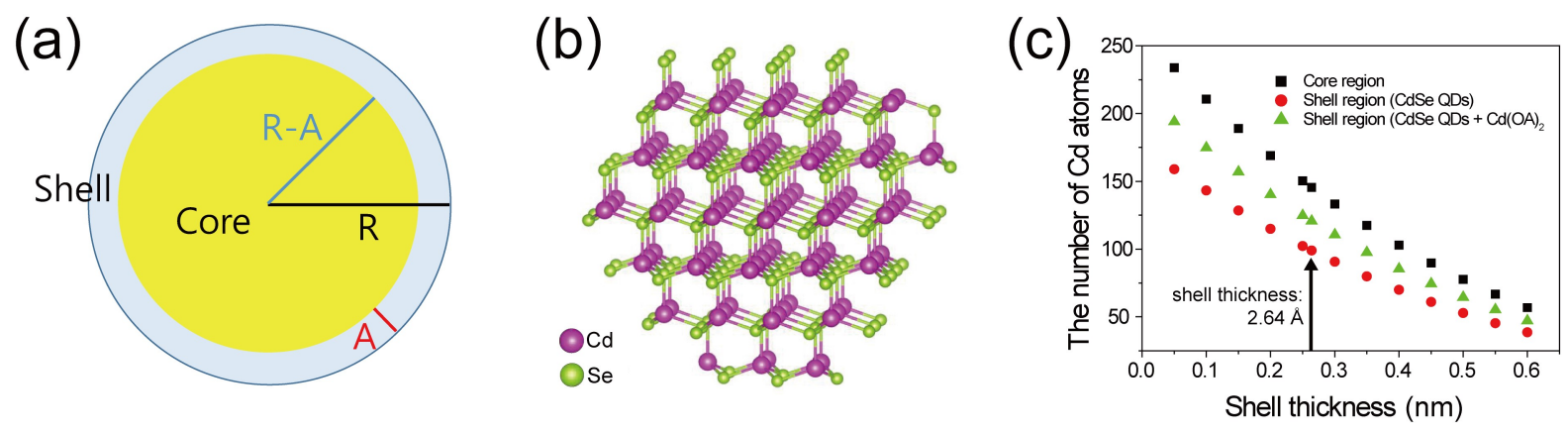

Figure S9. Schematics of the CdSe QD model from the Weiss group ${ }^{52}$ having $\mathrm{Cd} / \mathrm{Se}=1$ in the core and $\mathrm{Cd} / \mathrm{Se}>1$ in the shell (a) and the wurtzite structure CdSe QD core region illustrated with the VESTA software $^{54}$ (b). The QD was drawed with Cd and Se atoms within a radius (core region) of $2.490 \mathrm{~nm}$ from the center point. The plot of the number of $\mathrm{Cd}$ atoms according to the shell thickness (c).

In brief,

$\frac{\mathrm{Cd}}{S e}=\frac{V_{\text {core }}\left(\rho_{\text {cd,core }}\right)+V_{\text {she }}\left(\rho_{\text {Cd,shell }}\right)}{V_{\text {core }}\left(\rho_{\text {Se,core }}\right)+V_{\text {Shell }}\left(\rho_{\text {Se,she }}\right)}$

$\rho_{\text {Se,shell }}=0$ and $\rho_{C d, \text { core }}=\rho_{\text {Se,core }}$ where $\rho$ is a number density.

$\frac{\mathrm{Cd}}{S e}=1+\frac{\left(\frac{4 \pi R^{3}}{3}-\frac{4 \pi(R-A)^{3}}{3}\right)\left(\rho_{\text {Cd,shell }}\right)}{\frac{4 \pi(R-A)^{3}}{3}\left(\rho_{\text {Se,core }}\right)}=1+\frac{\left(R^{3}-(R-A)^{3}\right)\left(\rho_{\text {Cd,shell }}\right)}{(R-A)^{3}\left(\rho_{\text {Se,core }}\right)}$ 
To calculate the number of $\mathrm{Cd}$ atoms in the shell, we have rearranged the equation from the Weiss group for $\rho_{\text {cd,shell. }}$

$\rho_{\text {Cd,shell }}=\frac{(R-A)^{3}\left(\rho_{\text {Se,core }}\right)}{\left(R^{3}-(R-A)^{3}\right)}\left(\frac{\mathrm{Cd}}{S e}-1\right)$

$\mathrm{R}$ is calculated from TEM images (Table S1). We obtained density information using the CIF file of the wurtzite CdSe crystal (COD ID: 9016056) having a unitcell size of $112.1976 \AA^{3}$ (Figure S9b). In addition, the crystal model was fitted to the size obtained from the TEM image. The shell thickness A has been considered as the longest length $(0.264 \mathrm{~nm})$ of Cd-Se bonding (Figure S9c) because the Weiss model assumes that there are Cd atoms only in the shell. The result indicates that a CdSe QD has 98.9 Cd atoms in the shell, whereas one QD in the CdSe QDs $+\mathrm{Cd}(\mathrm{OA})_{2}$ sample has $120.7 \mathrm{Cd}$ atoms in the shell. 


\section{Calculation S2. The ligand composition of QDs.}

The $\mathrm{Cd} / \mathrm{Se}$ ratio could be measured with ICP-AES, and the numbers of $\mathrm{Cd}$ and Se atoms could be found by building up a QD model based on the $\mathrm{Cd} / \mathrm{Se}$ ratio. Using the ratio of $\mathrm{Cd}: \mathrm{Se}: \mathrm{P}$ obtained from ICPAES data, the number of $\mathrm{P}$ atoms could be calculated. NMR data were used to determine the ratio of the carboxylic acid to the phosphonic acid bound to the QD surface. The integral value of the peak near $5.6 \mathrm{ppm}$ designates that two protons arise from the olefinic position of oleic acid. A sharp pick near 1.0 ppm indicates three protons of the terminal carbon.

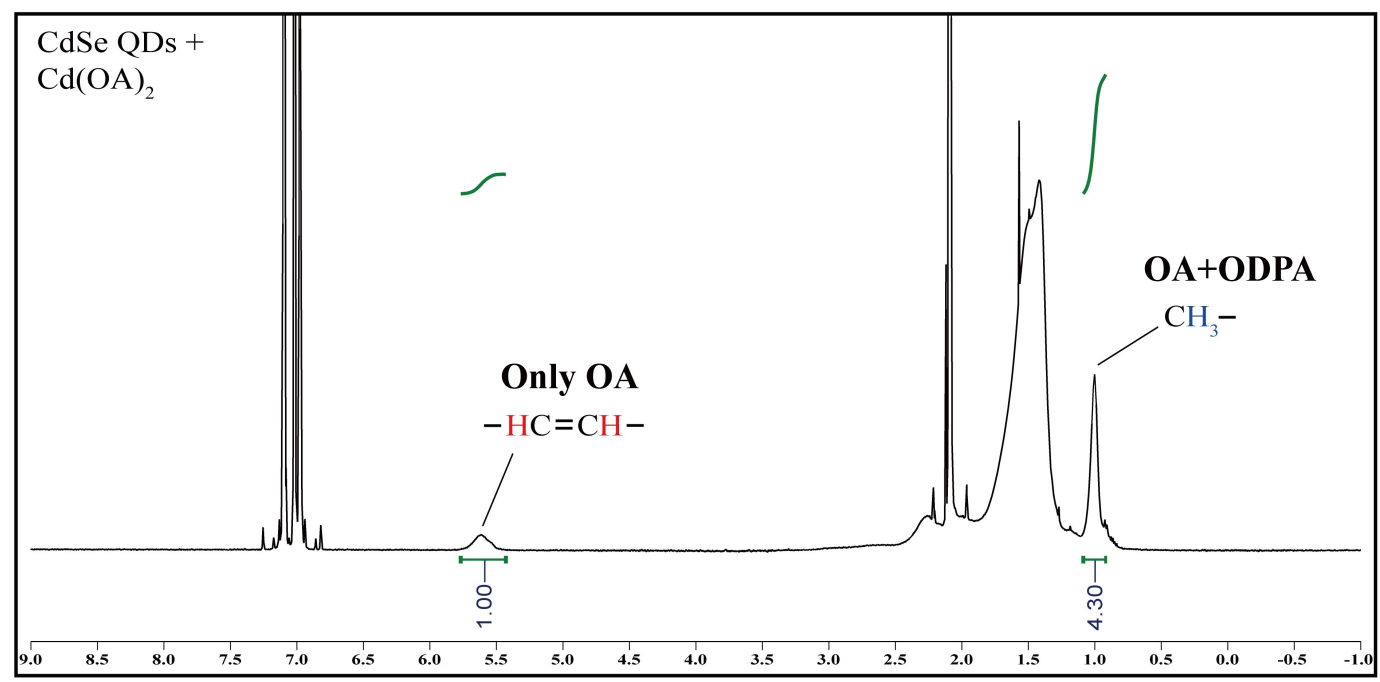

Figure S10. ${ }^{1} \mathrm{H}$ NMR spectrum of the $\mathrm{CdSe} \mathrm{QDs}+\mathrm{Cd}(\mathrm{OA})_{2}$ sample with the integrals of protons at the olefinic and the terminal positions of bound ligands.

We assign $\mathrm{A}$ to the number of OA molecules, $\mathrm{B}$ to the number of ODPA molecules, and $\mathrm{x}$ to the proportional constant of NMR integral values. According to our following simple calculation, we get 0.54 for the ratio of $\mathrm{A} / \mathrm{B}$.

$$
2 \mathrm{~A}=\mathrm{x}, \quad 3 \mathrm{~A}+3 \mathrm{~B}=4.3 \mathrm{x}, \quad \mathrm{A} / \mathrm{B}=0.54
$$

The number of ODPA molecules calculated from Calculation S1 indicates that 65.4 ODPA molecules were bound to the surface of QD in the CdSe QDs sample and that 52.4 ODPA molecules were attached to QD in the CdSe QDs $+\mathrm{Cd}(\mathrm{OA})_{2}$ sample. By multiplying the number of ODPA molecules with the 
OA/ODPA ratio, we find that 28.0 OA molecules were bound to QD in the CdSe QDs $+\mathrm{Cd}(\mathrm{OA})_{2}$ sample. 